\title{
Viable Tumor Diameter
}

National Cancer Institute

\section{Source}

National Cancer Institute. Viable Tumor Diameter. NCI Thesaurus. Code C96646.

The diameter of the portion of the tumor mass that is capable of growth, proliferation and metastasis. 